\title{
Evidences for a large hot spot on the disk of Betelgeuse ( $\alpha$ Ori)
}

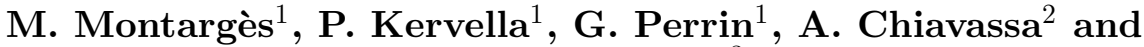 \\ J. B. Le Bouquin ${ }^{3}$ \\ ${ }^{1}$ LESIA, Observatoire de Paris, CNRS, UPMC, Université Paris-Diderot, 5 place Jules \\ Janssen, 92195 Meudon, France \\ email: miguel.montarges@obspm.fr \\ ${ }^{2}$ Laboratoire Lagrange, UMR 7293, Université de Nice Sophia-Antipolis, CNRS, Observatoire \\ de la Côte d'Azur, BP. 4229, 06304 Nice Cedex 4, France \\ ${ }^{3}$ UJF-Grenoble 1 /CNRS-INSU, Institut de Planétologie et dAstrophysique de Grenoble
}

(IPAG) UMR 5274, Grenoble, France

\begin{abstract}
Massive evolved stars contribute to the chemical enrichment of the Galaxy. When they die as supernova but also through their mass loss during the several thousands of years of their red supergiant (RSG) phase. Unfortunately the mass loss mechanism remains poorly understood. Detailed study of the CSE and photosphere of nearby RSGs is required to constrain this scenario.

Betelgeuse is the closest RSG (197 pc) and therefore has a large apparent diameter ( $\sim 42$ mas) which makes it a very interesting target. For several years, our team has lead a multi-wavelength and multi-scale observing program to characterize its mass loss. We will review here our recent results in near-infrared interferometry.
\end{abstract}

Keywords. stars: individual: Betelgeuse, supergiants, stars: late-type, techniques: interferometric, infrared: stars

\section{Introduction}

Massive stars contribute to the chemical evolution during the later stages of their existence through their spectacular supernova and in the quieter RSG phase. Material is moving away from the RSG thus it is cooling and forming molecules and dust that can reach the interstellar medium (ISM). Yet, several processes remain poorly understood. In particular we still do not know how the mass loss of RSG is triggered as these stars are not experiencing flares or pulsations like AGB stars. Josselin \& Plez (2007) suggested that huge convection cells could locally lower the effective gravity and levitate material in combination with radiative pressure on molecular lines. With the detection of a magnetic field around $\alpha$ Ori (Aurière et al. 2010), the hypothesis of the triggering of the mass loss by the dissipation of Alfvén waves (Airapetian et al. 2010) remains also an explanation.

As the closest RSG, Betelgeuse is the ideal target for observers to constrain the mass loss models. Hot spots were observed on this star by Haubois et al. (2009) with a reconstructed image in the $\mathrm{H}$ band from IOTA interferometric data and interpreted as a convective pattern by Chiavassa et al. (2010). More recently Ohnaka et al. $(2009,2011)$ observed upward and downward motions of CO in the MOLsphere with K band interferometry at high spectral resolution. 
Table 1. Best fitted value of the VLTI/AMBER data for the uniform disk (UD) and limb-darkened disk (LDD)

\begin{tabular}{c|ccc|}
\hline Model & $\theta$ (mas) & $\alpha$ & $\chi^{2}$ \\
\hline UD & $40.9 \pm 0.52$ & - & 110 \\
LDD & $41.8 \pm 0.57$ & $0.10 \pm 0.02$ & 55 \\
\hline
\end{tabular}

\section{VLTI/AMBER observations}

AMBER (Petrov et al. 2007, Astronomical Multi-BEam combineR, ) is installed at the ESO Very Large Telescope Interferometer (VLTI). It is combining the light from three telescopes in the $\mathrm{J}, \mathrm{H}$ or $\mathrm{K}$ band with spectral dispersion. Therefore it produces three observables : the visibilities, the differential and the closure phases. For a more detailed presentation of the interferometric techniques, see Van Belle's contribution to this conference.

We observed Betelgeuse with VLTI/AMBER with the Auxiliary Telescopes (ATs) of $1.8 \mathrm{~m}$ of diameter on January 1st, 2nd and 3rd and February 17th 2011. We used the medium spectral resolution of the instrument $(\mathrm{R}=1500)$ in the $\mathrm{H}$ band and in the $\mathrm{K}$ band (centered at $2.3 \mu \mathrm{m}$ ). The data reduction and the analysis of the $\mathrm{CO}$ and $\mathrm{H}_{2} \mathrm{O}$ absorption domain $(\lambda \in[2.245 ; 2.45 \mu \mathrm{m}])$ are presented in detail in Montargès et al. (2013). The data were reduced using amdlib 3.0.3, the AMBER reduction package $\dagger$. The stars HR 1543, HR 2275, HR 2469, HR 2508 and HR 3950 were observed as interferometric calibrators.

Here we will focus on the $\mathrm{K}$ band continuum data $(\lambda \in[2.1 ; 2.245 \mu \mathrm{m}])$. The data were fitted with two analytical models. First we used a uniform disk (UD) for which $I=I_{0}$ on the stellar disk and a power law limb-darkened disk (Hestroffer 1997) where $I=I_{0} \mu^{\alpha}$ with $\mu=\cos \theta$, the cosinus of the angle between the line of sight and the center of the disk. The results of these fits are presented in Table 1 . The visibilities together with the best fitted analytical model are plotted on Fig. 1.

If these analytical model reproduce well the first and second lobe of the visibility function (the $\chi^{2}$ falls to 5.27 and 4.28 for the UD and the LDD respectively when considering only those spatial frequencies while the best fitted parameters stay in their error bars), from Fig. 1 it appears that these simple models cannot reproduce the high spatial frequency signal which reveals the presence of smaller structures on the star photosphere.

To analyse the higher order lobes (spatial frequencies $>60 \operatorname{arcsec}^{-1}$ ) of the visiblity function and the closure phases, we used radiative hydrodynamics (RHD) simulations from the $\mathrm{CO}^{5}$ BOLD code (Freytag et al. 2012). The non-gray model st35gm03n13 was picked up (Chiavassa et al. 2011). The resolution of the grid was $235^{3}$ with a step of $8.6 R_{\odot}$. The parameters of the model are compared to those of Betelgeuse in Table. 2 .

From an initial homogeneous state the simulation is evolving. When the convection stationary regime is in place, several temporal snapshots are saved, each one becoming a realization of the convective pattern of the star. Intensity maps are produced with the 3D pure-LTE radiative transfer code OPTIM3D (Chiavassa et al. 2009) in each spectral channel of AMBER between 2.1 and $2.245 \mu \mathrm{m}$. As we do not know the orientation of the simulation relatively to the real star on the sky, we rotate each snapshot with a step of $10^{\circ}$. Thus we have a simulation grid of snapshots and rotation angle. The interferometric observables are then extracted from these maps (Chiavassa et al. 2010).

$\dagger$ Available at http://www.jmmc.fr/amberdrs 

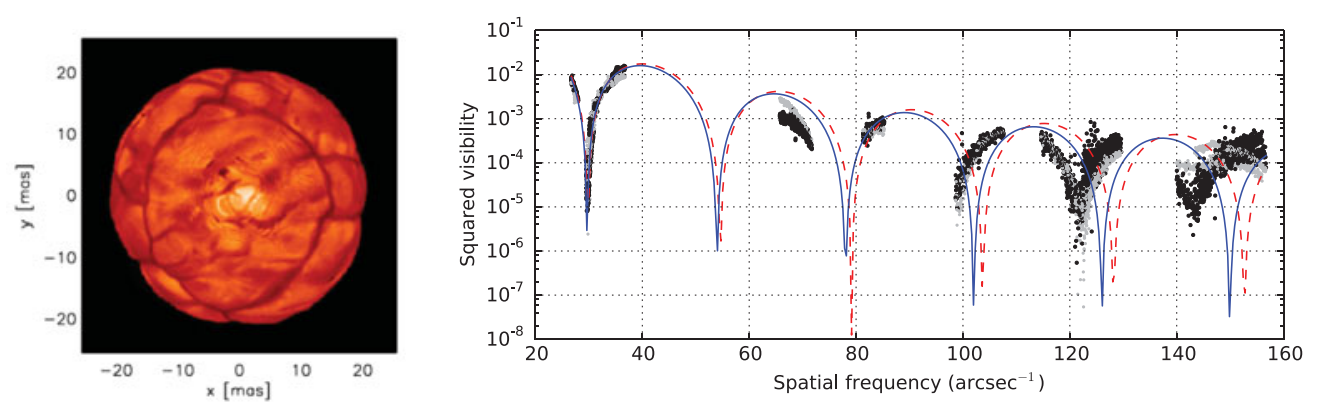

Figure 1. Best fitted snapshot of the RHD simulation. Left: Intensity map of the best fitted snapshot. Right: The black dots represent the squared visibilities of the VLTI/AMBER observations. The grey dots correspond to the best fitted snapshot. For comparison, the best fitted UD model is represented by the dashed line and the best fitted LDD model by the continuous line.

Table 2. Comparison between the physical caracteristics of Betelgeuse and of the RHD simulation.

\begin{tabular}{c|cc|}
\hline Parameter & Betelgeuse & Model \\
\hline$M\left(M_{\odot}\right)$ & $11.6(\mathrm{a})$ & 12.0 \\
$L\left(L_{\odot}\right)$ & $6.31 \times 10^{4}(\mathrm{~b})$ & $8.94 \times 10^{4}$ \\
$R\left(R_{\odot}\right)$ & $645(\mathrm{~b})$ & 846 \\
$T_{\mathrm{eff}}$ & $3640(\mathrm{c})$ & 3430 \\
$\log (g)$ & $-0.300(\mathrm{~d})$ & -0.354 \\
\hline
\end{tabular}

Notes:

(a): Neilson et al. (2011), (b): Perrin et al. (2004), (c) Levesque et al. (2005), (d) Harper et al. (2008).

We fitted the observed visibilities with the ones we derived from the RHD simulation. The best fitted snapshot and rotation angle give $\chi^{2}=7.47$ (See Fig. 1). Therefore the RHD simulation reproduces our VLTI/AMBER continuum observations better than the analytical UD and LDD model. However our closure phases are poorly reproduced (the $\chi^{2}$ goes up to 17 if we include the is observable). This means that the convective pattern we observe on our RHD simulation does not correspond exactly to the features on Betelgeuse's photosphere but that they match a convective signature. In other words, the structures observed with VLTI/AMBER in the K band on the photosphere of the star are clearly caused by convective cells but because of the uncertainties on their number, shape and position, we are unable to reproduce exactly their distribution within the actual observations and the use of this simulation. Only such statistical approach can validate the measured signal.

From the intensity map of the best fitted snapshot (Fig. 1), we notice that we do not observe giant convection cells or hot spots as observed by Haubois et al. (2009). The detailed study has been submitted to $A \mathscr{E} A$ (Montargès et al. 2014).

\section{VLTI/PIONIER monitoring of the photosphere}

We continued to observe Betelgeuse at the VLTI the following years. We gathered data with the new VLTI/PIONIER instrument on January 31th 2012, February 09th 2013 and January 11th 2014. PIONIER (Le Bouquin et al. 2011, Precision IntegratedOptics Near-infrared Imaging ExpeRiment, ) is a 4-telescopes instrument located at the Paranal observatory. It gives access to six visibilities and 3 independent closure phases 

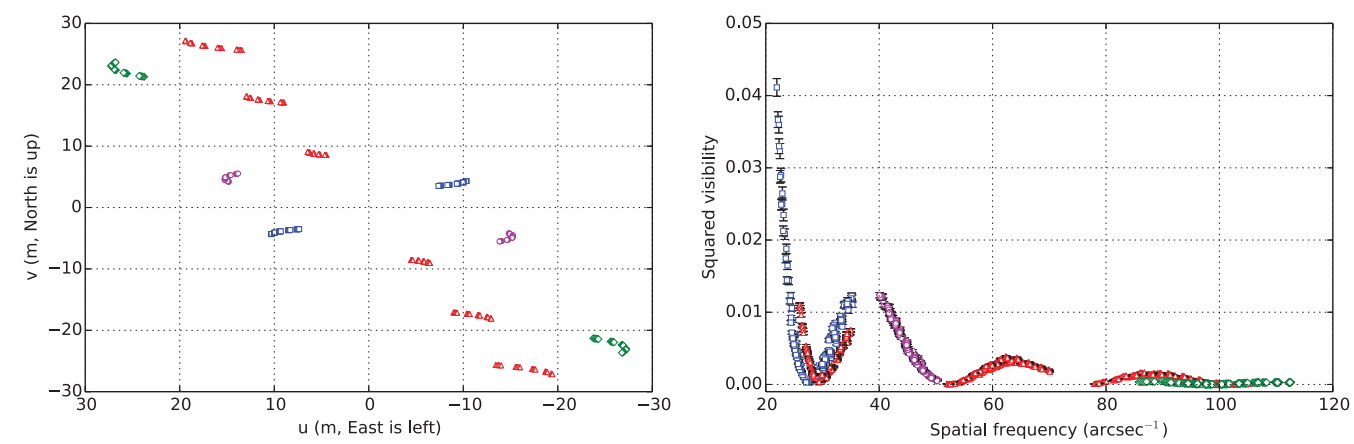

Figure 2. VLTI/PIONIER data of Betelgeuse of February 09th 2013 (see the online version of this proceeding for a color version of this figure). Left: $(u, v)$ plane of the observations with a symbol-coded PA. Right: Visibilities with matching symbols.

in one observation. It is a low spectral resolution instrument. We used it in the $\mathrm{H}$ band. Betelgeuse was observed with the four ATs in their compact array configuration. The data were reduced and calibrated using the pndrs package provided by the PIONIER team.

In 2012, Sirius was used as interferometric calibrator but is located $27^{\circ}$ away from Betelgeuse. As the interferometric transfer function of PIONIER is mainly sensitive to the distance between the science target and its calibrators and to avoid systematics we change our calibrators in 2013 and 2014 and used 56 Ori ( $6^{\circ}$ from Betelgeuse), b Ori $\left(7^{\circ}\right)$ and LTT $11688\left(5^{\circ}\right)$. Betelgeuse is too bright at infrared wavelengths, diaphragms were used in 2012 to lower the incoming flux. In 2013 we used a neutral density filter instead and in 2014 we lowered the exposure time by acquiring the data on three pixels of the $\mathrm{H}$ band (usually seven pixels are read)

For the three epochs the first zero of the visbility function occurs at different spatial frequencies along two directions of the $(u, v)$ plane (see Fig. 2 for an example on the 2013 epoch). This could indicate that the star has not the same size depending on the azimuthal direction probed. For the 2013 epoch, we can fit both direction on which we probe the first lobe by the power law limb-darkened disk described in Sect. 2. Counting $0^{\circ}$ in the North direction and $90^{\circ}$ in the East one, we have $\theta_{\mathrm{LDD}}=48.14 \pm 0.19$ mas for $\chi^{2}=46.89$ in the $32^{\circ}$ azimuthal direction (North-East) and $\theta_{\mathrm{LDD}}=44.54 \pm 0.32$ mas for $\chi^{2}=114.62$ at $113^{\circ}$ (South-East). Considering that Betelgeuse is $197 \mathrm{pc}$ away (Harper et al. 2008) this would cause a difference of stellar radius of $\sim 85 R_{\odot}$ or $7 \% R_{\star}$ if one consider the mean stellar radius. With the long rotational period of the star (Uitenbroek et al. 1998, $17 \mathrm{yr}$, ) the elliptical shape of the star is most likely unreal, moreover, it fails to reproduce the higher order lobes and gives a significant greater mean angular diameter than the 2011 observations (see Sect. 2).

Therefore, we adopted another model to fit these data : we used a power law limbdarkened disk as described earlier on which we added a gaussian hot spot characterized by its center ( $x_{\text {gauss }}^{\text {center }}, y_{\text {gauss }}^{\text {center }}$ ) its full width at half maximum (FWHM) and its flux weight relatively to the stellar disk flux. The results of these fits are presented on Table 3 and Fig. 3. This model reproduces the double first zero of the visibility function along the two direction of the $(u, v)$ plane and allows conserving a single angular diameter for Betelgeuse as expected (it also ensures continuity with the 2011 value). 
Table 3. Derived parameters from the fit of the limb-darkened disk and gaussian hot spot model to the VLTI/PIONIER data.

\begin{tabular}{|c|c|c|c|}
\hline Epoch & 2012 & 2013 & 2014 \\
\hline $\begin{array}{c}\theta_{\mathrm{LDD}}(\mathrm{mas}) \\
\alpha_{\mathrm{LDD}} \\
\text { Weight }_{\mathrm{LDD}}\end{array}$ & $\begin{array}{c}42.75 \pm 0.05 \\
0.13 \pm 0.01 \\
0.88 \pm 0.29\end{array}$ & $\begin{array}{c}43.47 \pm 0.05 \\
0.17 \pm 0.01 \\
0.82 \pm 0.24\end{array}$ & $\begin{array}{c}45.65 \pm 0.08 \\
0.37 \pm 0.02 \\
0.91 \pm 0.42\end{array}$ \\
\hline $\mathrm{x}_{\text {gauss }}^{\text {center }}$ (mas) & $19.0 \pm 0.2$ & $15.16 \pm 0.23$ & $18.71 \pm 0.53$ \\
\hline y yauss $\left._{\text {gauter }}^{\text {cenas}}\right)$ & $-7.23 \pm 0.23$ & $-2.79 \pm 0.21$ & $-20.37 \pm 0.41$ \\
\hline $\mathrm{FWHM}_{\text {gauss }}$ (mas) & $20.92 \pm 0.51$ & $24.73 \pm 0.31$ & $21.88 \pm 0.87$ \\
\hline $\begin{array}{c}\text { Weight }_{\text {gauss }} \\
\chi^{2}\end{array}$ & $\begin{array}{c}0.12 \pm 0.04 \\
61.13\end{array}$ & $\begin{array}{c}0.18 \pm 0.05 \\
86.48\end{array}$ & $\begin{array}{c}0.09 \pm 0.04 \\
97.22\end{array}$ \\
\hline
\end{tabular}

Notes:

The $x_{\text {gauss }}^{\text {center }}$ and $y_{\text {gauss }}^{\text {center }}$ are offsets from the star center. North and East are counted as positive offsets.
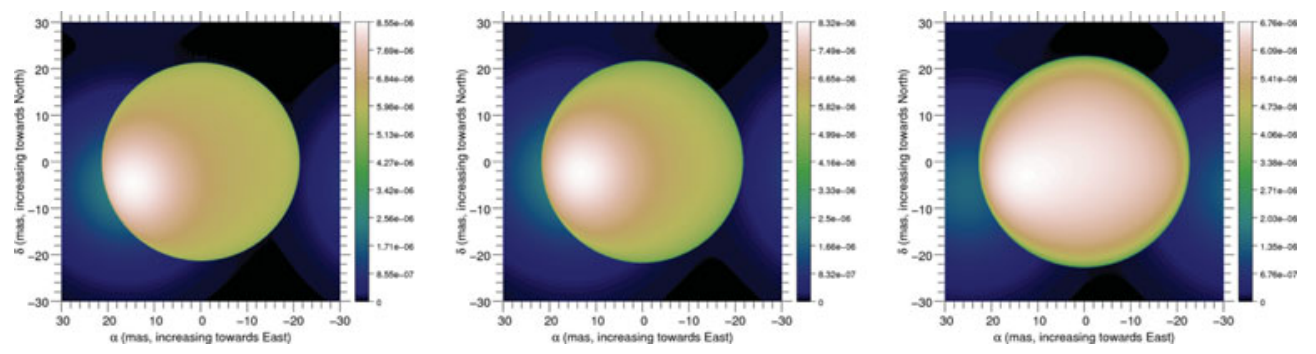

Figure 3. Intensity map of the best fitted model of the VLTI/PIONIER data by a limb-darkened disk and a gaussian spot. Left: January 2012. Center: February 2013. Right: January 2014.

\section{Conclusion}

VLTI near infrared data enabled us to monitor the photosphere of Betelgeuse, the closest RSG : we detected the signature of the convection with VLTI/AMBER in 2011 and monitor a hot spot (certainly a giant convection cell) with VLTI/PIONIER in 2012, 2013 and 2014. The VLTI/PIONIER model fitting will shortly be complemented by a projected spot on a sphere model (Baron et al. 2014) a RHD simulation analysis. These observations allow following the morphology and dynamics of the photosphere. Thanks to imaging at larger scale at different wavelengths (UV, radio), we will have a consistent view of Betelgeuse's CSE and a complete simultaneous snapshot of its mass loss which will strongly constrain the models.

\section{Acknowledgements}

This work is based on AMBER and PIONIER observations made with ESO Telescopes at the Paranal Observatory under programmes ID 086.D-0351, 286.D-5036(A), 288.D5035(A), 090.D-0548(A), 092.D-0366(A) and 092.D-0366(B). This research received the support of PHASE, the high angular resolution partnership between ONERA, Observatoire de Paris, CNRS and University Denis Diderot Paris 7. We acknowledge financial support from the Programme National de Physique Stellaire (PNPS) of CNRS/INSU, France.

\section{References}

Airapetian, V., Carpenter, K. G., \& Ofman, L. 2010, ApJ 723, 1210 
Aurière, M., Donati, J.-F., Konstantinova-Antova, R., et al. 2010, A $\& A$ 516, L2

Baron, F., Monnier, J. D., Kiss, L. L., et al. 2014, ApJ 785, 46

Chiavassa, A., Freytag, B., Masseron, T., \& Plez, B. 2011, A $\& A$ 535, A22

Chiavassa, A., Haubois, X., Young, J. S., et al. 2010, A\&A 515, A12

Chiavassa, A., Plez, B., Josselin, E., \& Freytag, B. 2009, A\&GA 506, 1351

Decin, L., Cox, N. L. J., Royer, P., et al. 2012, A\& A 548, A113

Freytag, B., Steffen, M., Ludwig, H.-G., et al. 2012, Journal of Computational Physics 231, 919

Harper, G. M., Brown, A., \& Guinan, E. F. 2008, AJ 135, 1430

Haubois, X., Perrin, G., Lacour, S., et al. 2009, A\& A 508, 923

Hestroffer, D. 1997, A\& A 327, 199

Josselin, E. \& Plez, B. 2007, A\&A 469, 671

Le Bouquin, J.-B., Berger, J.-P., Lazareff, B., et al. 2011, A\& $A$ 535, A67

Levesque, E. M., Massey, P., Olsen, K. A. G., et al. 2005, ApJ 628, 973

Montargès, M., Kervella, P., Perrin, G., \& Ohnaka, K. 2013, in P. Kervella, T. Le Bertre, \& G.

Perrin (eds.), EAS Publications Series, Vol. 60 of EAS Publications Series, pp 167-172

Montargès, M., Kervella, P., Perrin, G., et al. 2014, A\&AA, Submitted

Neilson, H. R., Lester, J. B., \& Haubois, X. 2011, in S. Qain, K. Leung, L. Zhu, \& S. Kwok (eds.), Astronomical Society of the Pacific Conference Series, Vol. 451, p. 117

Ohnaka, K., Hofmann, K.-H., Benisty, M., et al. 2009, A $\& A$ 503, 183

Ohnaka, K., Weigelt, G., Millour, F., et al. 2011, A\&SA 529, A163

Perrin, G., Ridgway, S. T., Coudé du Foresto, V., et al. 2004, A\&A 418, 675

Petrov, R. G., Malbet, F., Weigelt, G., et al. 2007, A\&SA 464, 1

Uitenbroek, H., Dupree, A. K., \& Gilliland, R. L. 1998, AJ 116, 2501

\section{Discussion}

ARNETT: How many zones does the 3D simulation have? Numerical resolution makes a difference and even heroic calculations can be unresolved.

MONTARGÈs: The resolution of the simulation is $235^{3}$. Indeed, as with every simulation the resolution constrains a lot the result. Increasing the numerical resolution brings more details in the structure but the overall convection is still there (ie., the large granules). We can try to increase to very high number the resolution but, for sure, we are still far from fully resolving all the convective structures from small to large cells.

Domiciano DE SouzA: Maybe to guide you in the model comparisons for Betelgeuse you could use analytical models with spots to have an idea of how the star looks like.

MontARGès: That's what we are trying to do : constraining the biggest spot with improved model fitting (spot on the limb of a sphere instead of a limb-darkened disk + gaussian spot model) and then, use this best fitted model to constrain the "first order" of the RHD simulation which will provide in addition the smaller structures. This is not an easy task though. It works in principle if the model spots have the right shape and if the number of spots is correct. The difficulty is that the high frequency data cannot be fully filtered as closure phases are a mix of different ranges of frequency. Rejecting all closure phases with a frequency larger than a threshold leaves with fewer low frequencies than what has been measured by the interferometer. Hence the difficulty sometimes to compare the low frequency fit with the image reconstructed using all the set of data.

LOBEL: Could you give us an estimate of the formation height of the H-band continuum in alpha Ori's extended atmosphere? Do you expect to find more surface spots when looking in the V-band, or at the formation level of photospheric absorption lines? Note that the UV sport observed in 1998 was imaged in the "chromosphere" which extends 
over 100 times the photospheric radius. Would you think that the spots you see in the $\mathrm{H}$-band are physically linked to the features observed in the UV, for example due to plumes that protrude the chromosphere?

MONTARGÈs: We usually consider that the $\mathrm{H}$ band continuum is forming at $\tau=1$, meaning at the angular diameter measured for the star. Concerning the convection observed in the $\mathrm{V}$ band, the strong atomic and molecular electronic absorption should increase the opacity thus stimulating the convection. But spots are also created by temperature inhomogeneities and we should keep in mind that $\mathrm{M}$ stars have a lower flux in the visible. The counter part of the convection in the chromosphere is one of the motivation of our executed HST/STIS imaging program but to establish a strong link between the different scales, one would need to perform a long survey at different scales and wavelengths. Note that Chiavassa et al. (2011) suggested a strong contribution of the convection at shorter wavelengths.

MEYNet: Betelgeuse is a runaway star. I do not know whether the rapid movement of the star may be a cause for the differences in radius you obtain from different directions ? Could for instance the movement change the wind near the star and make some anisotropies in some wavelengths?

MontARGÈs: The bow shock observed by Decin et al. (2012) is several arcmin away from the star. At the local scale ( tens of mas for the photosphere) such motion has poor or even no effect. The dynamics is dominated by the mass loss and the gravity of the star.

STEE: Since you have hydro-simulations at small spatial resolution, have you tried to convolve your high-res images with the "beam" of the interferometer to see if you can reproduce the observations in the first visibility lobe?

MONTARGÈs: Indeed the simulations do not reproduce such a big hot spot on the RSG photosphere but the convolution should not solve the hot spot issue as the first lobe is well resolved by the interferometer (the VLTI compact configuration alone allows to explore the visibility function up to the fifth lobe)

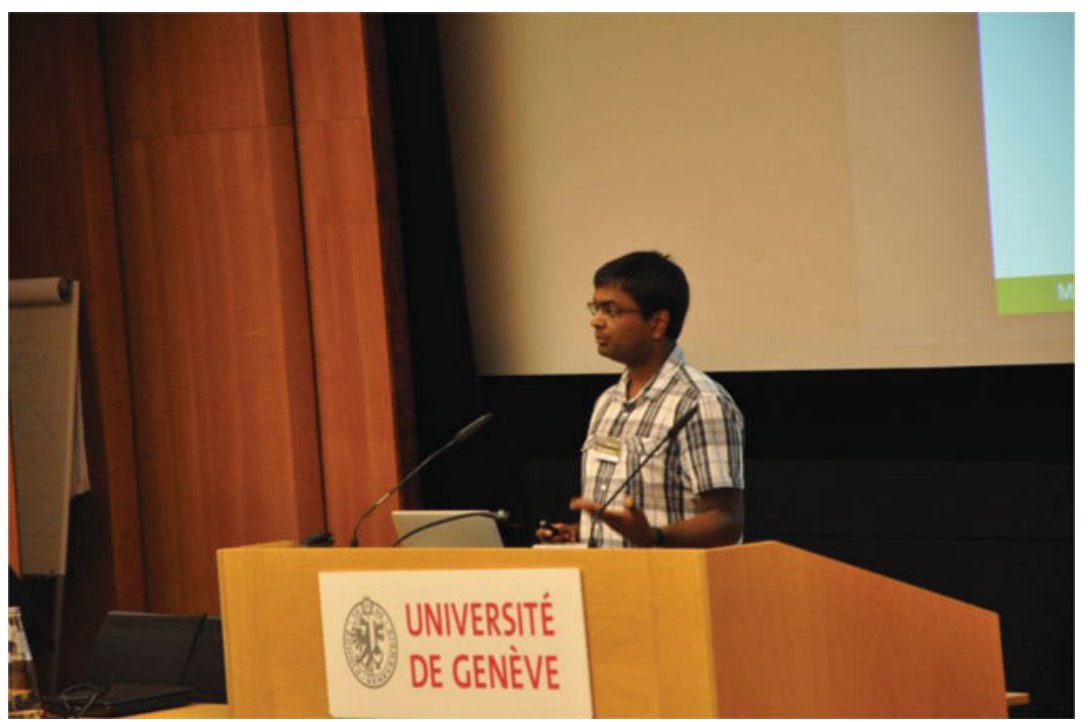

Miguel Montargès 